BRIEF COMMUNICATION

\title{
Malignant mixed mullerian tumour: A brief communication
}

\author{
Dibya S Malla, Achala Vaidya, Prabhaker Kumar
}

Maternity Hospital, Thapathali.

\begin{abstract}
Carcinosarcoma is a Malignant mixed mullerian tumor (MMT) originating from the mesothelium and mesenchyme of embryonic mesoderm, now better established by electron microscopy and immuno-peroxidase techniques.

The most interesting aspects in the histogenesis of this biphasic tumour mostly monoclonal in origin are briefly communicated in this paper. Unfortunately, no adjuvant chemo or radiotherapy for either persistent or recurrent malignant diseases has ever resulted in cure of this aggressive tumour, usually seen in menopausal women.
\end{abstract}

Key words: Malignant mixed mullerian tumor (MMT), carcinosarcoma, menopause.

\section{Introduction}

The malignant mixed mullerian tumor (MMT) is a neoplasm commonly encountered in the mullerian derivative of the female genital tract. It has an epithelial component; showing carcinomatous changes while mesenchyma, which shows the sarcomatous changes. So this is also called carsinosarcoma. Because of the combination of peritoneal epithelium and underlying mesenchyme, some have expressed it also as MMT.

With rare exception this is a disease of elderly menopausal women. ${ }^{1}$ This is a highly aggressive tumour and is diagnosed by histopathological examination of endometrial biopsy or hysterectomy specimen.

The main stay of treatment is surgery. In the management of these cases, no significant survival advantage was found when surgery is combined with radiation or chemotherapy. ${ }^{2}$ The interesting aspect of the origin of these biphasic malignant tumour, with a case report, is briefly communicated in this paper.

\section{Case}

A lady aged 74years complained of vaginal bleeding for three months following 19 years of menopause. Her katamenia was at the age of 19 years and delivered seven children. She was a small build lady and was not aneamic. Gynaecological examination revealed no vaginal bleeding and a bulky uterus. The ultrasonography showed an echogenic mass in the endometrial cavity of the uterus. Endometrial biopsy showed adenocarcinoma. Two months later total hysterectomy and bilateral salpingooopherectomy was carried out. During operation the uterus was of about 10 weeks size and was very soft. On section the cavity was full of fleshy polypoidal growth (masses) and two of them were attached to the uterus with a pedicle. She was discharged from hospital after smooth postoperative recovery.

Histopathology commented, on the specimen received as a uterus with both adenexas and omental tissue where the endometrial cavity contained grayish brown friable tissue masses, two of them being attached to the uterus by short pedicle.

The sections from uterine masses showed malignant neoplasm composed both (fig 1,2) of epithelial and mesenchymal elements involving one half of myometrium. The epithelial components were arranged in the papillae and tubules (fig 2). These malignant cells exhibited moderate cellular polymorphisms and nuclear atypia. Nuclei were hyperchromatic with coarsely granular chromatin pattern. Nucleoli were

\section{Correspondence}

Prof. D.S Malla. FRCOG

Maternity Hospital, Thapathali.

Email:dibyanm@healthnet.org.np 


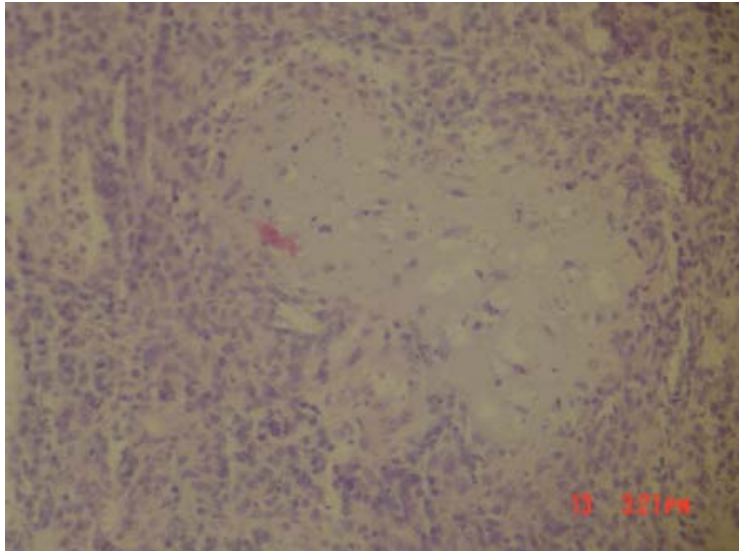

Fig. 1. Malignant Cartilage

prominent. The malignant mesenchymal element was in the form of cartilages (fig 1). Mitosis in the epithelial component was 4-8 / HPF. Extensive necrosis (fig 3) was identified. Vascular invasion was present. The cervix, both ovaries and tubes are free from tumour infiltration. The section from omentum showed no tumour cell infiltration. Impression: Carcinosarcoma (Malignant Mixed Mullerian tumour) involving half of the myometrial wall.

\section{Discussion}

Although Malignant Mixed Mullerian tumour (MMT) occurs infrequently, their clinical feature and diverse spectrum of histopathological pattern has been a subject of interest. The disease is manifested mostly with postmenopausal bleeding per vaginum. Some present polypoidal mass in the vagina or enlarged uterus as abdominal mass. This neoplasm has been associated with history of radiation therapy and some developed this malignancy while taking Tamoxifen. ${ }^{3}$ Clinical course of the disease is that of a high grade aggressive malignant tumour. One third of the patients have initial clinical evidence of extrauterine spread, and $40-50 \%$ of the stage I cases are found to have higher stage of disease at surgery. This woman was diagnosed

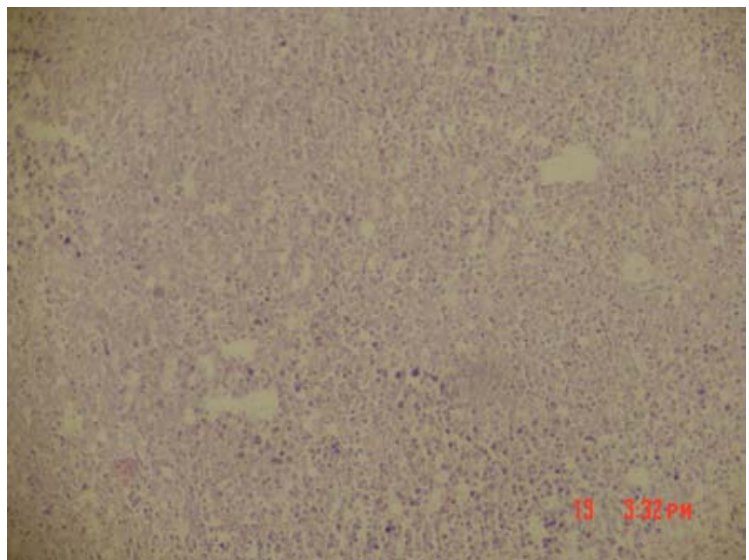

Fig. 3. Extensive Necrosis

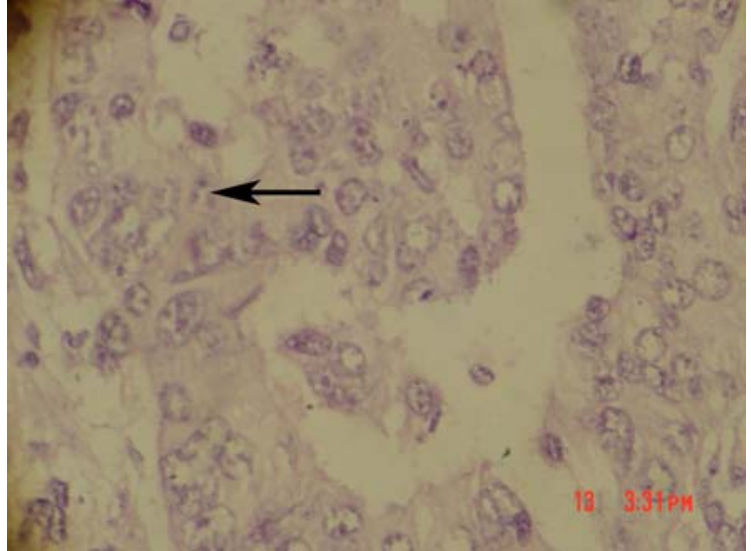

Fig. 2. Malignant Epithelial Component

as a case of endometrial carcinoma and postoperatively hysterectomy revealed stage II disease involving half of the myometrium. The histopathological reported proved it to be carcinosarcoma. The histology of endometrial biopsy specimen re-examined also reconfirmed carcinosarcoma.

No adjuvent therapy has been proved effective in a randomized trial by Gynaecology Oncology Group (GOG). Treatment of persistent or recurrent tumour by chemotherapy or radiotherapy hardly ever resulted in cure. $^{1}$

About half a century ago (1954) malignant mixed mullerian tumour had interested Gynecologist and Pathologist alike and they have reported twenty such cases in publication 'Cancer'. ${ }^{4}$ The peritoneal surfaces are host to a range of body organs and neoplasia in them. It is so because of the remarkable Mullerian potential of peritoneal mesothelium and adjacent mesenchyme. A study on 21cases of mixed mesodermal tumour revealed the origin of the tumour from the mesoderm of the Mullerian system and it was called Malignant mixed mesodermal mullerian tumour (MMMT). It reiterate the shared mesodermal ancestry with primary mullerian system which derives it from coelomic epithelium. ${ }^{5}$ Earlier it was found that different cell types in one tumour is not uncommon. These changes are explained as tumour metaplasia. This is more often seen in mesothelial tumours like that of the lung. About a fifth of lung adenocancinomas show squamous elements. Tumour cells can not be regarded as a completely differentiated tissue, so these variations in cell type is known as tumour metaplasia. The concensus of opinion is that the change process succeeds neoplasia much more than preceding it. ${ }^{6}$

During first half of this period of interest in carcinosarcoma (1960-1980) much of the literature commented on difficulties and inconsistencies in the terminology of this tumour. Primary peritoneal mullerian tumors have been hypothesized to arise from the mesothelium and sub-mesothelial mesenchyme through 
a process of metaplasia. Since similar neoplasia have been described in men, these lesions, from the so called "Secondary Mullerian System" a term coined by SC Lauchlan in 1972, are not restricted to female peritonium. Extragenital MMMT with neuroendocrine differentiations has been reported by Cokelaere $\mathrm{K},{ }^{7}$ it was arising from jejunal mesentry and was composed of a poorly differentiated epithelial component and spindle cell component with heterologous (rhabdomyoblastic) differentiation. In a case report of uterine-like mass consistent with an extrautrine adenomyoma was found in the pelvic peritonium of 22 years of following a total hysterectomy and bilateral salpingo-oopherectomy. Thus they concluded, most likely the uterine like mass to arise from the tissues of the secondary mullerian system in response to estrogenic stimulation. ${ }^{8}$ Another case was reported in a woman aged 83 years where MMT with heterologous element was arising from the caecal peritoneum. ${ }^{9}$

By 1980s onwards the wide spread use of electron microscopy (EM) and immunohistochemistry clarified the epithelial nature of these sarcomas. The epithelial components in carcimosarcoma are mucinous, squamous, endometrioid, high grade papilary, clear cell, undifferentiated or mixture of these types. It is traditional to divide stromal component into homologous (leomyosarcoma, stromal sarcoma and fibro sarcoma) and heterologous (chondrosarcoma, rhabdomyosarcoma, osteosarcoma and liposarcoma) types. Identification of heterologous sarcoma is often diagnostically useful in singling out the tumour as carcinosarcoma. The Electron Microscopy (EM) and Immuno-Peroxidase techniques (IPX) have been used to study the cellular detail of these (MMT) tumours. The IPX analysis included monoclonal antibodies to keratin (K) and Vimentin (V) and polyclonal antibody to myoglobin. Carcinomatous elements were always $\mathrm{K}+$ and homologous stromal sarcoma cells were V+. Ultrastructurally the epithelial cells were not highly differentiated and the sarcomatous elements generally resembled normal proliferate- phase stromal cells. ${ }^{10}$

Recently immunohistochemical cell culture and molecular genetic studies have been reported in a publication - Frequent genetic heterogeneity in the clonal evolution of gynaecological carcinosarcoma and its influence on phenotypic diversity. Just to mention there are three main hypotheses which exist to explain these diverse elements, the first one is a collision tumour theory, then the composition theory, and the last combination tumour theory. All favors these cancers to be monoclonal in origin. In addition, the extent of intratumoral genetic heterogenecity in these tumors with divergent histology has also been studied in detail by micro dissection. They found shared allelic loses and retention among multiple individually dissected foci in 17 cases. The results strongly support the monoclonal origin for this neoplasia (carcinosarcoma). They have genetic progression and diversion parallel the development of divergent phenotype. This divergence most likely occurs relatively late in the evolution of the tumor. ${ }^{11}$

In summary, carcinosarcoma is a mixed mullerian tumor originating from the mesothelium and mesenchyme of embryonic mesoderm. These tumours from primary and secondary mulerian system are monoclonal with few exceptions. Some genetic hetrogenecity to accompany the phenotypic divergence within individual tumor also have been demonstrated. The combination of tumour hypothesis implies a common stem cell precursor with two phenotypically different cell populations in the histogenesis of malignant mixed mullerian tumor and the sarcomatous component arises in a carcinoma through evolution of the sub clones.

Mostly the tumour is seen in menopausal women, the clinical course is that of highly aggressive malignant nature. The histogenesis of the tumor has been better established with the development of modern technology.

\section{Acknowledgment}

The authors would like to thank Ms Gyanu Rana for her valuable help in preparing the article.

\section{References}

1. Mills SE, Carter D, et. al. Carcinosarcoma : In Sternbyrg's Diagnostic Surgical Pathology, $4^{\text {th }}$ Edition 2004 : 2494 - 2495.

2. Nielsen SN. Podratz KC. Scheithauer BW, O'Brien PC. Clinicopathological ananlysis of uterine malignant mixed mullerian tumours. Gynecol Oncol 1989Sep; 34(3): 372-378.

3. Mccluggage, W.G. Abdulkadert M. Price.J.H, .Kelhan P, Hamilton S, J Beattie, Al-Nafussi A. Uterine Carcinosarcoma in patients receiving tamoxifen. A report of 19 cases. Int j Gyynecol Cancer 2000; $10: 280-284$.

4. Sternberg SG, Clark WH, Smith RC. Malignant mixed mullerian tumour (mixed mesodermal tumor of the uterus): A study of twenty one cases. Cancer 1954; 7 :704-724. 
5. Silverberg SG, Major FJ, Blessing JA, Fetter B, Askin FB, et. al. Carcinosarcoma (malignant mixed masodermal tumour) of the uterus. A Gynaecology Oncology Group pathologic study oh 203 cases. Int J Gynecol Pathol 1990; 9 (1): 1-19.

6. Walter J.B and Talbot IC. Metaplasia: Abnormalities of cellular differentiation, In Walter and Israel General Pathology. $7^{\text {th }}$ Edition. Churchill Livingston, London. 1996 :418-422.

7. Cokelaere K, Michielsen P, De Vos R, Sciot R. Primary Malignant Mixed Mesodermal (Mullerian) tumour with Neuroendocrine Differentiation . Mod Pathol $2001 ; 14(5)$ : 515-520

8. Redman R, Wilkinson EJ, Massoll NA. Uterinelike mass with feature of an extrauterine adenomyoma presenting in 22 Years after total hysterectomy- bilateral salpingo-oophorectomy: A Case Report with review of the literature Arch Pathol Lab Med 2005 ; 129 (8 ) :1041-1043.

9. Mira JL, Fenogilo-Preiser CM, Husseinzadeh N. Malignant mixed mullerian tumour of extraovarian secondary mullarian system. Arch Pathol Lab Med; 1995, 119 (11): 1044-1049.

10. Geisinger KR, Dabbs DJ, Marshal L. Malignant Mixed Mullerian tumour: An ultrastructural and immunohistochemical Analysis with histogenetic consideration Cancer. 1987; 59: 1781- 1790.

11. Hiroika F, Manabu $\mathrm{Y}$ et al. Frequent genetic heterogeneity in the Clonal evolution of gynecological carcinosarcoma and its influence on phenotypic diversity. Cancer Research 2000; $1: 114-120$ 